\title{
Habituation and sensitization in primary headaches
}

\author{
Gianluca Coppola ${ }^{1 *}$, Cherubino Di Lorenzo ${ }^{2}$, Jean Schoenen ${ }^{3}$ and Francesco Pierelli ${ }^{4}$
}

\begin{abstract}
The phenomena of habituation and sensitization are considered most useful for studying the neuronal substrates of information processing in the CNS. Both were studied in primary headaches, that are functional disorders of the brain characterized by an abnormal responsivity to any kind of incoming innocuous or painful stimuli and it's cycling pattern over time (interictal, pre-ictal, ictal). The present review summarizes available data on stimulus responsivity in primary headaches obtained with clinical neurophysiology. In migraine, the majority of electrophysiological studies between attacks have shown that, for a number of different sensory modalities, the brain is characterised by a lack of habituation of evoked responses to repeated stimuli. This abnormal processing of the incoming information reaches its maximum a few days before the beginning of an attack, and normalizes during the attack, at a time when sensitization may also manifest itself. An abnormal rhythmic activity between thalamus and cortex, namely thalamocortical dysrhythmia, may be the pathophysiological mechanism subtending abnormal information processing in migraine. In tension-type headache (TTH), only few signs of deficient habituation were observed only in subgroups of patients. By contrast, using grand-average responses indirect evidence for sensitization has been found in chronic $T \mathrm{TH}$ with increased nociceptive specific reflexes and evoked potentials. Generalized increased sensitivity to pain (lower thresholds and increased pain rating) and a dysfunction in supraspinal descending pain control systems may contribute to the development and/or maintenance of central sensitization in chronic TTH. Cluster headache patients are chrarcterized during the bout and on the headache side by a pronounced lack of habituation of the brainstem blink reflex and a general sensitization of pain processing. A better insight into the nature of these ictal/interictal electrophysiological dysfunctions in primary headaches paves the way for novel therapeutic targets and may allow a better understanding of the mode of action of available therapies.
\end{abstract}

Keywords: Migraine; Tension-type headache; Cluster headache; Trigeminal autonomic cephalalgias; Sensitization; Habituation; Evoked potentials; Reflex; Pain mechanisms; Thalamocortical dysrhythmia

\section{Review Introduction}

Among the general population, headaches are highly prevalent and receive growing attention not only because they affect people's quality of life, but also because they have a significant economic impact. Idiopathic or primary headache syndromes are disorders in which there is a temporary or permanent dysfunction of the central nervous system, often genetically determined, without apparent organic lesion. They include migraine, tension

\footnotetext{
* Correspondence: gianluca.coppola@gmail.com

'Department of Neurophysiology of Vision and Neurophthalmology, G.B. Bietti Foundation IRCCS, Via Livenza 3, 00198, Rome, Italy

Full list of author information is available at the end of the article
}

headache, and the trigeminal autonomic cephalalgias among which "cluster headache". Progress in headache research has benefited from the International Classification of Headache Disorders (ICHD), and its revisions [1,2], because they have provided operational diagnostic criteria allowing for a better comparison of clinical data between headache centres.

In the recent publication of the Global Burden of Disease survey 2010, tension-type headache and migraine are the second and third most prevalent disorders in the world, and migraine is recognized as the seventh highest cause of disability in the world [3]. The large and still growing scientific knowledge on their pathophysiological mechanisms has contributed the recognition

\section{它}


of headaches as neurological conditions worth of interest. In fact, although scientists have not completely disentangled the complicated puzzle of primary headache pathophysiology, great advances were made during the last 2 decades with the help of the new research armamentarium.

Clinical neurophysiology methods, in particular, have allowed in vivo measurements of the headache patients' electrocortical responses to various sensory stimuli. They are atraumatic, non-invasive and complementary to modern neuroimaging techniques, and thus suitable to study functional disorders as primary headaches.

In their episodic forms, primary headaches are defined as paroxysms (the attacks) separated by remissions of variable lengths. Several studies focused therefore for ictal versus interictal electrophysiological abnormalities, in order to understand the predisposition and the recurrent character of attacks. In this respect, among the various primary headaches, migraine is doubtless the beststudied headache type.

Migraineurs are characterized interictally by a generally increased sensitivity to visual (sensitivity to light), auditory (to sound), or somatic stimuli (allodynia) not only during the attack, but also outside of the attack. Researchers, trying to explain this phenomenon, observed that interictally migraineurs with and without aura show a time dependent amplitude increase of scalp-evoked potentials to repeated stereotyped stimuli with respect to normal subjects. This phenomenon was called "deficient habituation" and was only seen during the painfree period for almost all sensory modalities. However, this is not a static phenomenon, but, as shown in several studies, habituation changes with the proximity to an attack, during the attack and when episodic migraine evolves to chronic migraine, a complication of migraine where sensitization, the opposite of habituation, makes its appearance, changing fundamentally the response pattern.

The present article, after providing an overview of the general concept of habituation and its opposite "sensitization", will review the studies on habituation/ sensitization in migraine and other primary headaches, performed with different clinical neurophysiology methods and emphasize in particular the more recent data.

\section{General concept}

Habituation is defined as "a response decrement as a result of repeated stimulation" [4] and is a common feature of responses to any kind of sensory stimulation. It is an ubiquitous phenomenon observed in different experimental settings and in neuronal circuits of a wide range of complexity, from the withdrawal reflex of the gill and siphon in Aplysia to the autonomic and behavioral component of the whole-of-body reflex called the "orienting response" in humans [5-7].

Habituation is a multifactorial event of which the accompanying synaptic plastic mechanisms are still not totally elucidated. Several theories, or at least hypotheses, have been proposed over the years to explain this phenomenon [8]. In the 70's, Groves and Thompson proposed the "dual-process" theory, stating that two separate and opposing processes, depression (habituation) and facilitation (sensitization), compete to determine the final behavioural outcome after a sequence of repetitive stimuli [6]. According to the dual-process theory, sensitization is the side of the pendulum that, when present, prevails at the beginning of the stimulus session and accounts for the initial transitory increase in response amplitude, whereas habituation occurs later during the course of the recording session and accounts for the delayed response decrement [6]. At the synaptic level, the stimulus-response pathway interacts with an external "state" system represented by various "tonic" non-specific and motivational circuits, including the ascending reticular activating system and related structures. In humans, these structures comprise the monoaminergic nuclei in the brainstem, that are critically involved in the central processing of arousal, control of the signal-to-noise ratio generated by sensory stimuli at cortical and thalamic levels, and endogenous antinociception [9].

To avoid semantic misunderstanding, it should be noted that a response dishabituation does not refer to lack of habituation, but to a response sensitization or "heterosynaptic facilitation" as termed by Kandel and associates in their works on Aplysia [10]. In fact, dishabituation is an actual recovery of the habituated response caused by the interference of an unexpected stimulus markedly different from the habituating ones. Sensitization is an elementary form of behavioral plasticity, perhaps equal in importance to habituation and apparently generated by somewhat different neuronal mechanisms [11].

Habituation depends on a series of parametric properties or characteristics [5], which were revised and refined during a workshop in Vancouver in 2007 [12]. These characteristics dealt with both short- as well as longterm habituation and its opposite dishabituation.

The phenomenon of habituation is considered useful for studying the neuronal substrates of behavior, the mechanisms of learning processes, or information processing in the CNS both in health and in disease.

It must be noted that, during the last three decades, sensitization has assumed a wider meaning than habituation. Sensitization is not only considered a general behavioral response of augmentation to innocuous sensory stimuli, but it has also acquired a particular significance 
in the field of pain as an augmentation of sensory signalling in the central nervous system as a consequence of noxious stimulation (for a review see Woolf, 2011 [13]). The latter may be a peripheral injury activating smallfiber afferents that produce an increase in excitability, i.e. sensitize, nociceptive spinal cord neurons. This increase in excitability is responsible for the plastic changes in those neural structures belonging to the socalled "pain matrix". It results in decreased nociceptive thresholds and increased responsiveness to noxious and innocuous peripheral stimuli, as well as expansion of the receptive fields of central nociceptors [14]. Overall, this transient or persistent state of higher reactivity is called "central sensitization". Studies in the headaches field disclosed changes in pain sensitivity that were interpreted as reflecting central sensitization. A wellrecognized clinical expression of central sensitization is cutaneous allodynia, which is prevalent during episodic migraine attacks $[15,16]$ and in chronic migraine [17-19]. There is no common agreement yet about what causes and where starts the cascade of events that lead to central sensitization in migraine or in other primary headaches. However, some evidences point towards sequential sensitization of first-order or second-order trigeminovascular nociceptors via overt (aura) or possibly silent (without aura) cortical spreading depression waves or, more likely, via an indirect activation of pain modulatory structures in the brainstem (raphe magnus, locus coeruleus and other aminergic nuclei) and the forebrain (periaqueductal gray, rostroventral medulla) $[20,21]$.

In human research, time-locked cortical potentials (EP) evoked by a sensory stimulation and reflex responses (RR) after electric stimulation of a peripheral nerve have been frequently used to study the habituation and sensitization phenomena. A common way of analysing these responses consists of either analysing single trials or averaging in blocks single epochs of EP or RR per subject, identifying the latencies and amplitudes or areaunder-the-curves of the considered components, and using these parameters as a dependent variables in the statistical analysis. Data are usually expressed either in absolute or log-transformed values, and between single trials or blocks habituation is calculated either with linear regression (slope of the linear regression line) or with block ratios (change in amplitude expressed in percentage).

\section{Habituation in migraine}

Neurophysiological data suggest that lack of habituation during stimulus repetition despite an initial normal or slightly lower response amplitude is a functional, probably genetically determined, property of the brain in migraineurs between attacks.
The very first study showing that habituation is decreased in patients affected by migraine without aura between attacks was carried out with contingent negative variation (CNV), a slow negative cortical response related to higher mental functions [22-26]. The CNV abnormality was more evident for the early than for the late component [24,27-33], and was assessed by visual $[34,35]$ or auditory [36-38] oddball paradigms. The lack of habituation was confirmed several times by analyzing visual evoked potentials (VEP) in response to checkerboard pattern [39-49], and also by using magneto electroencephalography [50-52]. The abnormal visual information processing in migraine seems to be characterized by an initial response of normal or slightly lower amplitude followed by an amplitude increase, that, as stated above, corresponds neither to response sensitization nor to dishabituation, but to a lack of habituation [40-50]. The same phenomenon was also found with somatosensory [53-55] and auditory [56,57] evoked cortical potentials in migraine between attacks. Sand et al. (2008) recorded brainstem auditory evoked potentials (BAEPs) in migraine interictally and found lack of habituation in the wave IV-V [58], a datum that was recently confirmed also in a few migraineurs experiencing vertigo, especially when symptomatic [59]. Moreover, Sand et al. also observed a positive relationship between BAEP amplitudes and blood serotonin level in healthy controls, but not in migraine, a result that was interpreted as an evidence in support of the notion that a dysregulation of the serotonin system is linked to migraine pathogenesis [58].

Besides the innocuous evoked potentials, researchers have verified if the same habituation deficit might exist after noxious stimulation. The blink reflex (BR) obtained after supraorbital stimulation with a so-called "nociception-specific" electrode, a way to more specifically explore trigeminal nucleus caudalis activation, discloses an interictal habituation deficit during short $[60,61]$ as well as long time courses [44,62]. Amplitude and habituation of another brainstem reflex, the clickevoked vestibulocollic reflex, was equally reduced in migraineurs between attacks compared with healthy subjects $[63,64]$.

Brief radiant heat pulses, produced by $\mathrm{CO} 2$ laser stimulation, activate selectively $\mathrm{A} \delta$ and $\mathrm{C}$ fibres and generate an evoked potential that can be recorded from the temples (early component, N1) and the vertex (late components, N2-P2) of the skull. In episodic migraine the late component of laser evoked potentials (LEPs), mainly generated in the insular and anterior cingulate cortices, elicited by either cephalic (usually supraorbital) or extracephalic (usually hand dorsum) stimulation does not habituate during stimulus repetitions over short [65] or long durations [66-68]. Deficient habituation was also observed for the early N1 LEP component, mainly 
generated in the secondary somatosensory cortex $[66,69]$. In two studies performed in episodic migraine between attacks using contact-heat evoked potentials (CHEPS) and source localization with standardized LORETA (sLORETA) mapping it was shown that the lack of habituation to the noxious heat stimuli is probably related to the inability of the orbitofrontal cortex (OFC) to filter out correctly the pain information $[70,71]$. Since evidence from animal and human studies suggests a role for serotonin in the OFC-mediated descending inhibition of pain [72], the authors argue that a possible alteration of brain 5-HT neurotransmission may be responsible for this abnormal pain information processing in migraine, where decreased serotonergic disposition was previously reported [73-75],

Genetic load seems to play an important role in the mechanisms that produce physiologically altered habituation. In fact, the habituation deficit in migraineurs has a family character. This abnormality is present interictally not only in adults, but also in children in whom it is significantly correlated with that of their parents $[28,76]$. Moreover, asymptomatic subjects defined to be "at risk" for developing migraine, i.e. healthy but with first-degree relatives with migraine, present the same habituation deficit in evoked potentials and nociceptive blink reflex as established migraineurs, so that deficient habituation can be regarded as neurophysiological marker of pre-symptomatic migraine [29,62].

Since migraine is a recurrent paroxysmal disease characterized by attacks (ictal period) and variable pain-free periods (interictal), it was of interest to perform sequential recordings during the days preceding the attacks, immediately before or during the attack. During the days preceding the attack (pre-ictally) VEP and SSEP amplitudes increase and habituate normally [50,54,58,77,78], whereas CNV and P300 habituation is minimal and amplitude peaks $[30,79,80]$, suggesting that, depending on sensory modality, the habituation deficit worsens interictally, reaches its maximum a few days before the attack and then normalizes during the attack.

Finally, it must be mentioned that among more than fifty positive studies, the habituation deficit in migraine during the pain-free phase was not confirmed in some studies $[77,81-87]$. It is not easy to explain why some research groups did not retrieve any habituation deficit in migrainous patients between attacks. For some Authors lack of blindness for diagnosis during the recording sessions may be an explanation [87], but the same research group did not find lack of habituation even with no blindness [77]. Another one could be the use of different patients' selection criteria, such as recruitment of university students or medical staff instead of patients who spontaneously visited a Headache Clinic, the latter experiencing more day life discomfort from their migraine.
Whatever the explanation, we must take into account that habituation deficit is not constant in migrainous patients. In fact, habituation degree may change not only interictally vs. pre-ictally vs. ictally, but also within the pain-free period with the distance since the last or next attack [88]. Moreover, specific genetics influence [76,89] and clinical fluctuations, such as spontaneous clinical worsening or improving of attacks frequency [90,91], may vary the baseline level of thalamocortical activation [92] and then the degree of habituation in migraine [55].

\section{Mechanisms of the habituation deficit in migraine}

As mentioned above, the neural mechanisms underlying habituation remain poorly understood, and this uncertainty helps to explain why the abnormal habituation pattern in migraine still lacks a definitive consensual interpretation [93-95].

Neuromodulatory techniques, like repetitive transcranial magnetic stimulations (rTMS) and transcranial direct current stimulations (tDCS), were used to shed more light on the interictal abnormal information processing in migraine. In migraineurs activating high frequency rTMS over the visual or somatosensory cortices was able to increase for some minutes the amplitude of the first VEP and somatosensory evoked potentials (SSEP) block and to normalize habituation over successive blocks, whereas inhibiting low frequency rTMS had negligible effects on both. By contrast, in healthy volunteers, inhibiting rTMS reduced first VEP and SSEP amplitude block as well as habituation, while the activating protocol had no effect $[42,55]$. A longer lasting effect (several weeks) on VEP was induced with 5 consecutive daily sessions of inhibiting rTMS over the visual cortex in healthy subjects, while the effect of activating rTMS in migraineurs lasted only hours or a few days [45]. Recently, Viganò et al. (2013) applied another activating neuromodulation method, anodal tDCS over the visual area in migraineurs, and reported that, similar to $10 \mathrm{~Hz}$ rTMS, the the 1st VEP block increased in amplitude and habituation normalized [96]. In the second phase of their study, the same authors performed a preventive pilot trial with 2 sessions of anodal tDCS over the visual cortex per week for 8 weeks in 15 migraine patients and found a clear beneficial effect on several clinical endpoints up to an average of 4.8 weeks after the tDCS treatment period [96]. Overall, the neuromodulatory studies indicate that only procedures that enhance cortical excitability are able to normalize the abnormal interictal information processing in migraine.

Further information on the pathophysiology of the interictal dysfunction in migraine was obtained from the more sophisticated studies of the high-frequency oscillations (HFOs) embedded in common somatosensory and visual evoked potentials. Early somatosensory HFOs, 
reflecting spike activity in thalamo-cortical cholinergic drives, were decreased interictally in migraine and normalized during the attack while late HFOs, reflecting primary cortical activation, were normal [97] or decreased [98]. Moreover, the reduction early HFOs was associated with a worsening in the clinical course of migraine [90]. In a recent study in migraineurs activating rTMS over the sensorimotor cortex was able to increase the interictal low thalamo-cortical drive. This was not the case in healthy volunteers probably because their thalamo-cortical activity was already maximal before the rTMS [97]. This finding supports the hypothesis that deficient habituation in migraine is due to a reduced thalamic control of the activity in sensory cortices i.e. a low pre-activation level. Further evidence for an abnormal thalamic control in the migraine brain intericatlly comes from the analysis of visual HFOs (gamma-band oscillations, GBO) [46]. We demonstrated a significant habituation deficit of the late $\mathrm{GBO}$ components in migraineurs relative to healthy subjects, which we interpreted as indicative of a dysfunction in cortical oscillatory networks that could in turn be due to an abnormal thalamic pacemaker rhythmic activity, namely "thalamo-cortical dysrhythmia" [46]. The latter may reconcile the long-lasting controversy between excessive excitation and deficient inhibition in migraine, since a deficient thalamo-cortical drive, i.e. a low level of cortical preactivation, results in dysfunction of both inhibition and excitation. Lower inhibition and preactivation may thus co-exist, since the latter can promote the former via reduction of lateral inhibition [93]. Refined VEP techniques have shown that it is possible to enhance the relative contributions that arise from short- and long- range lateral inhibition between neurons through differential temporal modulation of adjacent regions of radial windmill-dartboard (W-D) or partial-windmill (P-W) visual patterns [99-101]. This may represent a further tool for investigating migraine pathophysiology. According to our recent study, the degree of short-range lateral inhibition in the visual cortex during W-D visual stimulation is more pronounced in migraine patients than in healthy volunteers at the beginning of the stimulus session (1st block). Over successive blocks of recordings, however, it decreases in migraineurs, but remains unchanged in healthy controls. During the migraine attack, short-range lateral inhibition is on the contrary much reduced, but it increases during stimulus repetition. There was no significant between group difference in the P-W amplitude, reflecting longrange lateral inhibition, and its attenuation [88]. These results favour a migraine cycle-dependent imbalance between excitation and inhibition in the visual cortex that results in a heightened cortical response to repeated stimuli, i.e. a lack of habituation. We hypothesized that an interictal hypoactivity of monaminergic pathways may cause a functional disconnection of the thalamus in migraine leading to an abnormal intracortical shortrange lateral inhibition, which could contribute to the habituation deficit observed during stimulus repetition. That in migraine the thalamus abnormally controls the cortex via thalamorcortical loops is further underscored by the recent study of the paired associative stimulation (PAS) paradigm, a protocol that uses in humans a design principle very similar to those producing long-term depression (LTD) or potentiation (LTP) in animal studies [102-104]. In migraine, depressing PAS paradoxically increased motor evoked potential amplitudes instead of decreasing them, and enhancing PAS induced only a slight non significant response potentiation [105]. This suggests that impaired long-term associative synaptic plasticity mechanisms characterize migraine without aura patients between attacks. Because we observed, at least in a subgroup of subjects, that the PAS-induced plastic changes were inversely related with thalamocortical activation, as assessed by early somatosensory HFOs, we suggested that the malfunction in PAS-induced effects in migraine might reflect low cortical preactivation, which prevents short-term and longer-term changes in cortical synaptic effectiveness [105].

\section{Sensitization in migraine}

The majority of the studies on the dynamic behavior of peripheral reflexes and evoked cortical responses in migraine have focused on habituation instead of sensitization, probably because it is particularly difficult to assess sensitization by calculating "sliding" averages using successive blocks of few responses.

However, if sensitization, defined as facilitation occurring at the beginning of the stimulus presentation, is impaired in migraine (i.e. enhanced in comparison to habituation), it should be detectable as a higher first block absolute amplitude value of the considered response.

Indirect signs of sensitization were observed during migraine attacks and in chronic migraine patients with or without medication overuse.

It was reported several times that within the 12-24 hours preceding the attack the habituation pattern of reflex and non-noxious evoked potentials normalize. This has been shown with CNV $[26,79,80]$, VEP $[50,78,88]$, visual P300 latency [35], and nociceptive blink reflexes [60].

Pain-related responses may behave in a partially different way. During migraine attacks, the area-under-thecurve of the nociceptive blink reflex R2 component is temporary increased on the affected side in comparison with the non-affected side was observed [106]. Similar results were obtained using another noxious stimulation, the radiant laser $\mathrm{CO} 2$ : amplitude of the $\mathrm{N} 2-\mathrm{P} 2$ complex at the vertex was increased on the 
affected side compared to the non-affected side [107-109]. Interestingly, in episodic migraine LEPs did not habituate not only interictally, but also during the attacks, underscoring the different cerebral processing of noxious versus innocuous stimuli. These data could represent the electrophysiological counterpart of central sensitization of cerebral structures belonging to the so-called "pain matrix" that seems to be related to the mechanism of the migraine attack and of the chronification of migraine. As abovementioned, from a physiological point of view, sensitization refers to the plastic changes in neural structures belonging to the "pain matrix" that result in decreased nociceptive thresholds and increased responsiveness to noxious and innocuous peripheral stimuli, and expansion of the receptive fields of CNS nociceptive neurons [14]. As a matter of fact, reduced pain thresholds have been found clinically with quantitative sensory testing immediately before [110] and during [16] a migraine attack, but sometimes even earlier in the interictal period in some $[111,112]$ but not all the studies [110,113-115]. During attacks of migraine, reduced cutaneous pain thresholds on both symptomatic and non-symptomatic sides are accompanied by significantly increased N2-P2 complex of LEP [107] and changes in its dipolar source localization [108]. These abnormalities worsen with the increase in attack frequency $[107,108]$. In a group of chronic migraine (CM) patients, a trend for an increase in LEP N2P2 amplitudes [116] and a reorganization of the cortical areas devoted to pain processing [117], was also detected. Moreover, in CM due to medication overuse LEP N2-P2 amplitude still showed reduced habituation after both hand and face stimulation, similarly to the response behavior found with LEPs during interictal and ictal periods [118]. Interestingly, withdrawal from the acute medication overuse normalized the habituation curve [118].

Recently, somatosensory evoked potentials (SSEPs) proved to be ideal for disclosing sensitization (reflected by an increased response amplitude to low numbers of stimuli) and habituation (reflected by a decrease in response amplitude after high numbers of stimuli) in both episodic and chronic forms of migraine. While SSEPs confirmed a lower initial amplitude and late abnormal habituation in migraineurs studied interictally [53,54], a clear-cut sensitization, as reflected by a significant increase in SSEP 1st N20-P25 block amplitude, was found during an attack, followed by a normal habituation [54]. In medication overuse headache $(\mathrm{MOH})$ patients, we managed to record SSEPs in a pain-free state or during mild headaches and found that N20-P25 SSEP amplitude was initially (1st block) greater in $\mathrm{MOH}$ patients than in the subgroup of episodic migraineurs studied interictally and healthy controls [54]. The increased SSEP amplitude in $\mathrm{MOH}$ was proportional to the duration of headache chronification. We interpreted these results as reflecting reinforcement and perpetuation of central sensitization due to the medication overuse and increased headache frequency [54]. We further noted that the presence of such sensory sensitization depends on the class of drugs overused, since initial SSEP amplitudes were smaller in triptan overusers than in NSAIDs or combined overusers. The abnormalities in cortical responses to somatosensory stimulation seem to be strongly influenced by genetic factors. $\mathrm{MOH}$ patients carrying the D/D polymorphic variant of the angiotensin converting enzyme (ACE), that plays a role in neural plasticity and dependence behaviour, showed less SSEP habituation, in proportion with the duration of the overuse headache, and increased sensitization depending on the overused drug compared to I carriers [89].

Sensitization in migraine patients who evolved to chronic daily headache due to medication overuse was also demonstrated with pain-related evoked potentials (PREPs). Ayzenberg and co-workers recorded PREPs after electrical stimulations of cephalic (forehead) and extracephalic (hand dorsum) sites with a nociceptionspecific electrode. They observed a significant increase in PREP amplitudes both after cephalic and extracephalic stimulations in all patients with $\mathrm{MOH}$ irrespective if they overused NDAIDs or triptans. Withdrawal from the acute medication overuse normalized the PREP amplitude [119].

Sensitization phenomena might also manifest themselves at the spinal level. Perrotta and coworkers explored the spinal cord pain processing by studying threshold, area and temporal summation threshold (TST) of the lower limb nociceptive withdrawal reflex in a group of 31 $\mathrm{MOH}$ patients before and after acute drug withdrawal. A significantly lower reflex threshold, higher amplitude and lower TST was found in $\mathrm{MOH}$ patients before detoxification in comparison with episodic migraine and controls [120]. All these neurophysiological abnormalities tended to improve after a detoxification program [120], which was coupled with an increased activity of the endocannabinoid system [121].

\section{Habituation in tension-type headache}

There are only a few reports about habituation in tension-type headache (TTH).

No habituation deficit was observed with visual evoked or event-related potentials in episodic [41] or chronic TTH patients $[34,41]$. Episodic TTH sufferers had normal habituation of P300 latency, while P300 amplitude also showed some degree of habituation, although not of statistical significance [122].

Patients affected by chronic TTH showed a normal reducing behavior (habituation) in scalp potentials evoked by $\mathrm{CO} 2$ laser stimulation (LEPs) of the hand and facial 
skin [66]. Mismatch negativity, which is believed to reflect the automatic central processing of a novel stimulus, and P300 habituation were significantly lower in migraine and TTH children than in healthy subjects in one study, where P300 habituation also positively correlated with behavioral symptomatology [123]. In TTH, habituation was also investigated using sympathetic skin responses (SSR), a tool used to evaluate autonomic dysfunction. Ozkul and Ay explored SSR changes with the same stimulus at a constant intensity by four blocks of 20 responses and found that in both episodic migraine without aura and TTH patients there was a lack of habituation compared to normal controls [124]. The electrophysiological similarities between the episodic forms of migraine and TTH support the hypothesis that some patients with TTH might be at the mild end of the migraine spectrum. Since lack of habituation was not always observed in TTH, it seems to be relevant only for a subgroup of patients.

\section{Sensitization in tension-type headache}

In $\mathrm{TTH}$ and in cluster headache, like in migraine, the vast majority of neurophysiological studies have only indirectly assessed sensitization, through the measure of the grand-average area under the curve or amplitude of the given test.

The few studies in which the dynamic behavior of responses was analyzed using successive blocks of a few averagings were unable to find clear evidence for sensitization, i.e. for increased amplitude of the first block. This was the case in episodic TTH for VEPs [41], visual P300 [122], LEPs [123] and sympathetic skin responses [124], in chronic TTH for visual P300 [34], and LEPs [66].

By contrast, some indirect evidence for sensitization was found in $\mathrm{TTH}$, chiefly in its chronic form, with nociceptive specific reflexes and evoked potentials.

Normal amplitude, area, latency [125-128] and slower recovery cycle [127] of the blink reflex R2 component was found in chronic TTH. Two separate groups found reduced latencies of the trigemonocervical reflex in patients with chronic TTH [129-131]. Using a nociceptionspecific electrode lower values of the normalized root mean square and area under the curve of the blink with control subjects [132].

More convincing evidence for central sensitization in CTTH has come from studies of pain sensitivity in pericranial or lower limb tissues. Sandrini et al. (2006) studying the nociceptive lower limb flexion RIII reflex found significantly lower subjective pain thresholds and RIII reflex threshold in chronic TTH than in controls [115]. These findings were associated with a paradoxical facilitation of the RIII reflex response during the cold pressor test, which indicates deficient descending inhibition, an abnormality also found by others [133]. Previous studies have found normal pressure pain thresholds (PPTs) in episodic TTH $[134,135]$. In chronic TTH instead, PPTs were decreased [15,136,137] especially on the anterior part of the temporalis muscle [15,135-139] and in the upper part of the trapezius muscle [140]. Cathcart et al. (2010) investigated temporal summation, defined as the increase in pain perception to repeated noxious stimulation (indirect measure of sensitization), by an algometer and heterotopic noxious conditioning stimulation (HNCS) in chronic TTH vs. controls. Pain from repeated algometer pressures increased more in the CTTH sufferers compared with healthy controls, both at finger and shoulder, and was less inhibited by conditioned HNCS [141]. Lower pain thresholds in muscle and skin of the cephalic region but not of the extracephalic region with higher rating to suprathreshold single and repetitive $(2 \mathrm{~Hz})$ electrical stimulation were reported in patients with chronic TTH than in healthy controls [142].

In a LEP study, the heat pain threshold was similar in chronic TTH patients and controls at the level of both the hand and pericranial skin. The total tenderness scores (TTS) at pericranial sites were higher in TTH patients than in controls. The amplitude of the N2a-P2 LEP complex elicited by stimulation of the pericranial zone was greater in TTH patients than in controls and this was significantly associated with the TTS score [143].

\section{Habituation in cluster headache and other trigeminal autonomic cephalalgias (TACs)}

During the last decades, great advance in the understanding of the cluster headache pathophysiology was made with the modern techniques of functional neuroimaging [144]. Electrophysiological methods contributed to the study of cognitive and nociceptive processes.

Normal cognitive habituation was found in two visual event-related potential studies in cluster headache either during the bout or outside, and in chronic paroxysmal hemicrania [34,145].

Formisano et al. were the first to found abnormal habituation of the blink reflex in a small number of $\mathrm{CH}$ patients during the attack, but comparison with control subjects was lacking [146]. Habituation of both the R2 and the R3 blink reflex components are impaired in $\mathrm{CH}$ patients on the affected side compared to healthy controls [147]. The lack of habituation in $\mathrm{CH}$ patients was even more pronounced than that found in episodic migraine [147]. We recently replicated these results by using the nociception-specific concentric stimulating electrode: R2 reflex area and habituation were reduced on the affected $\mathrm{CH}$ side (data published in abstract form [148]). Conversely, Holle et al. (2012) failed to detect 
altered habituation of the nBR R2 in episodic and chronic $\mathrm{CH}$ within or outside a bout. In the latter study, however, the majority of $\mathrm{CH}$ patients were taking one or several prophylactic medications at the time of recordings, which may biased the results [149].

\section{Sensitization in cluster headache and other trigeminal autonomic cephalalgias (TACs)}

Classical blink reflex studies did not disclose any sign of sensitization in $\mathrm{CH}[150,151]$. In 10 episodic cluster headache patients within a bout, Lozza et al. (1997) found a significantly faster R2 blink reflex recovery curve on the symptomatic side after paired supraorbital stimuli, probably reflecting an indirect sign of sensitization within the spinal trigeminal nucleus. Furthermore, in the same study the R2 recovery curve was faster on both affected and unaffected sides in $\mathrm{CH}$ patients when the supraorbital stimulus was preconditioned by a peripheral stimulation of the index finger. Since naloxone injection transiently reverted this bilateral R2 sensitization, the authors postulated that the faster R2 recovery reflects hypoactivity of reticular nuclei, due to reduced descending opiatergic inhibition [152], a mechanism that was recently supported by functional neuroimaging studies $[153,154]$.

The threshold of the corneal reflex was reduced on the affected side in a mixed group of episodic (during the bout) and chronic $\mathrm{CH}$ patients, and normalized in the remission phase [155]. Others were not able to confirm such lateralized abnormalities.

Researchers found that both in- and out-side the bout patients had lower thresholds for pressure pain [156], electric pain and RIII reflex [157] on the affected than on the unaffected side both in episodic (in and outside of a bout) and chronic $\mathrm{CH}$ [158]. These signs of sensitization within the nociceptive system were coupled with a phase shift of the normal circadian rhythmic variations in RIII threshold in episodic bouts of $\mathrm{CH}$ when compared with the remission period, and with absence of circadian rhythmicity in chronic $\mathrm{CH}$ patients [158]. Perrotta et al. (2013) recently studied the functional activity of the descending diffuse noxious inhibitory controls (DNIC) (or conditioned pain modulation system) elicited by a cold pressor test (CPT) in a group of episodic $\mathrm{CH}$ patients during active and remission phases. Compared to healthy subjects, the RIII reflex threshold and TST were lower and the R2 area higher during, but not outside of a bout. $\mathrm{CH}$ patients during the bout had a significant reduction of TST compared both to controls and to $\mathrm{CH}$ patients outside of a bout. Only during the bout but not outside, the CPT had no effect on TST and reflex area [159]. The authors concluded that $\mathrm{CH}$ patients have a dysfunction of the supraspinal control of pain that depends on the clinical activity of the disease and leads to facilitation of pain processing predisposing to the $\mathrm{CH}$ attacks.

Further evidence for lateralized abnormalities came from the study of Procacci et al. (1989) who found cutaneous and deep hyperalgesia to both mechanical and electrical stimuli with earlier appearance of pain after an ischaemic test in the upper limbs on the affected side of the body in episodic $\mathrm{CH}$ patients [160]. By contrast, with quantitative sensory testing, perception of warmth, cold and pressure pain was reduced on the cluster side as compared with the contralateral asymptomatic side in a pooled group of episodic and chronic $\mathrm{CH}$ patients $[161,162]$; warm detection thresholds and thermal sensory limen on the affected side correlated negatively with elapsed time since last attack [162].

We are aware of only one study on sensitization in other trigeminal autonomic cephalalgias. In 12 patients with chronic paroxysmal hemicrania and 12 with hemicrania continua, pain pressure threshold, subjective pain perception after sural nerve stimulation as well as RIII reflex threshold were reduced mostly on the affected side, compared to healthy subjects [163]. Moreover, although there were no abnormalities in the blink reflex, corneal reflex thresholds were significantly reduced on both sides only in chronic paroxysmal hemicrania patients.

\section{Discussion}

Neurophysiological studies have disclosed various abnormalities of spinal, brainstem and cortical responsivity to external innocuous or noxious stimuli in primary headaches. These abnormalities can be summarized as follows:

- Abnormalities of the habituation/sensitization mechanisms were discovered in migraine. In episodic migraine, most published EP studies show two characteristic changes: a lack of habituation on recordings performed between attacks and sensitization during the attack, especially with somatosensory stimuli. The habituation deficit normalizes during attacks, whereas sensitizations vanishes between attacks, but in the immediate preictal phase both sensitization and deficient habituation may variably co-exist in response to non-noxious and pain stimuli. In patients who developed $\mathrm{MOH}$ the cortical response pattern could be locked in a pre-ictal state associating both initial sensitization and late deficient habituation, which contrasts with episodic migraine where these cortical states alternate (Figure 1). Recent works suggest that an abnormal rhythmic activity between thalamus and cortex, namely thalamocortical dysrhythmia, may be the pathophysiological mechanism subtending abnormal information processing in migraine. 


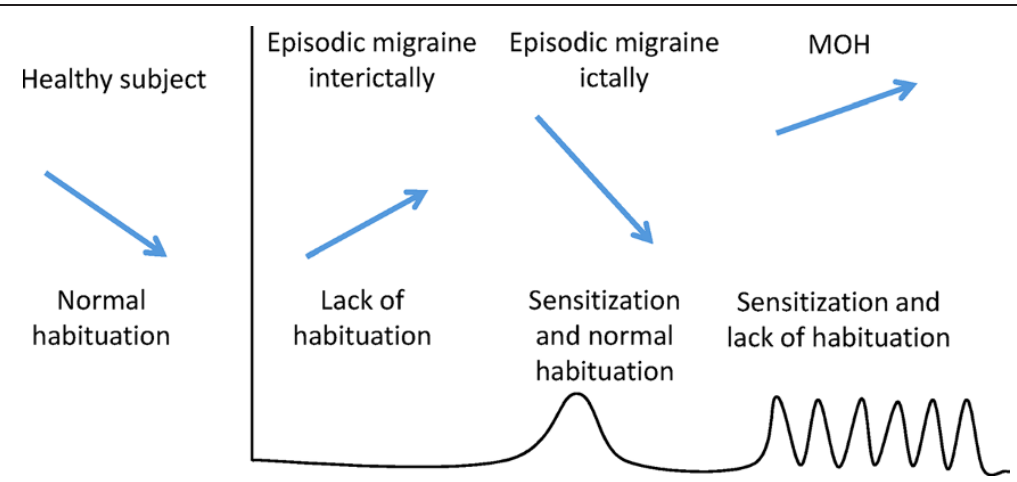

Figure 1 Schematic representation of the changes in habituation and sensitization in an healthy subject and over the migraine cycle (interictal, ictal, and chronic migraine due to medication overuse [MOH]).

- In tension-type headache, available studies are limited. Some, though only in subgroups of patients, found some evidence of deficient habituation, chiefly with cognitive potentials (mismatch negativity and P300) and sympathetic skin responses. By contrast, using grand-average responses indirect evidence for sensitization has been disclosed in chronic TTH with nociceptive specific reflexes and evoked potentials. These studies provide evidence for generalized increased sensitivity to pain (lower thresholds and increased pain ratings) and a dysfunction in supraspinal conditioned pain modulation in CTTH, which may contribute to the development and/or maintenance of central sensitization in this disorder.

- The deficient habituation of the blink reflex found in episodic $\mathrm{CH}$ patients during the bout suggests that interictal migraine and cluster headache probably share some pathophysiological mechanisms. However, the more pronounced habituation deficit found in the $\mathrm{CH}$ with respect to the migraine group suggests that additional dysfunctional neurobiological factors are at work in $\mathrm{CH}$ patients. Only during the bout but not outside, a sensitization of pain processing was observed. Several possible non-mutually exclusive causes could be responsible for this:

i) dysfunctioning descending aminergic, especially dopaminergic, control $[164,165]$, ii) malfunctioning hypothalamo-trigeminal control [166], and iii) altered descending opiatergic pain control system $[153,154]$. Given that $\mathrm{CH}$ patients had no habituation deficit of event-related potentials $[34,145]$, it is likely that the pathogenic factors involved in $\mathrm{CH}$ produce functional changes at the level of the trigeminal system but not at cortical level. Altogether, these data indicate that in cluster headache lateralised abnormalities may occur throughout the body, probably because of sensitization in the central nervous system and activation of nociceptive reflexes.

\section{Conclusions}

In migraine research, progress will largely depend on a better understanding of the mechanisms underlying the habituation deficit, its variations with the migraine cycle and its relation with changes in thalamo-cortical rhythms and brain stem-(thalamo-)cortical aminergic pathways. Future studies will have to determine whether there is an interaction between abnormal sensory processing and metabolic abnormalities, for instance decreased brain ATP content.

It will also be of importance to gather more data on the geno- phenotype correlations in migraine and in the other primary headaches. Such genotype/phenotype correlations could help to tailor treatment to the individual patient depending on his genetic profile [89].

In cluster headache, future electrophysiological works should try to understand the role of the descending monoamine and opioid systems in the mechanism of sensitization and lateralization of pain. Moreover, they may help to unravel the mechanisms that periodically ignite the cluster period and the culprits for the transformation of episodic into chronic $\mathrm{CH}$.

Finally, better characterizing headache patients from a neurophysiological point of view will allow to optimize the protocols of the minimally invasive techniques and non-invasive neurostimulation methods, and to improve their therapeutic efficacy $[96,167,168]$.

\footnotetext{
Abbreviations

BR: Blink reflex; $\mathrm{CH}$ : Cluster headache; CM: Chronic migraine;

CNV: Contingent negative variation; CPT: Cold pressor test; $\mathrm{CTTH}$ : Chronic tension-type headache; EP: Evoked potential; GBOs: Gamma-band oscillations; HFOs: High-frequency oscillations; HNCS: Heterotopic noxious conditioning stimulation; LEP: Laser evoked potential; $\mathrm{MOH}$ : Medication overuse headache; OFC: Orbitofrontal cortex; PAS: Paired associative stimulation; PREPs: pain-related evoked potentials; PPTs: Pressure pain thresholds; P-W: Partial-windmill; RR: Reflex response; rTMS: repetitive Transcranial Magnetic Stimulations; SSEP: Somatosensory evoked potential; SSR: Sympathetic skin responses; tDCS: transcranial Direct Current
} 
Stimulations; TST: Temporal summation threshold; TTH: Tension-type headache; TTS: Total tenderness score; W-D: Windmill-dartboard.

\section{Competing interests}

The authors declare that they have no competing interests.

\section{Authors' contributions}

GC made substantial contributions to review the literature as well as in drafting the manuscript. CDL, JS, and FP were implied in drafting the manuscript. All authors read and approved the final manuscript.

\section{Author details}

'Department of Neurophysiology of Vision and Neurophthalmology, G.B. Bietti Foundation IRCCS, Via Livenza 3, 00198, Rome, Italy. ${ }^{2}$ Don Carlo Gnocchi Onlus Foundation, Milan, Italy. ${ }^{3}$ Headache Research Unit, University Department of Neurology \& GIGA-Neurosciences, Liège University, Liège, Belgium. ${ }^{4}$ IRCCS Neuromed, Pozzilli (IS), Italy.

Received: 7 June 2013 Accepted: 21 July 2013

Published: 30 July 2013

\section{References}

1. Headache Classification Subcommittee of the International Headache Society (2004) The International Classification of Headache Disorders: 2nd edition. Cephalalgia 1:9-160

2. Olesen J, Bousser MG, Diener HC, Dodick D, First M, Goadsby PJ, et al. (2006) New appendix criteria open for a broader concept of chronic migraine. Cephalalgia 26:742-746

3. Martelletti P, Birbeck G, Katsarava Z, Jensen R, Stovner L, Steiner T, et al. (2013) The Global Burden of Disease survey 2010, Lifting The Burden and thinking outside-the-box on headache disorders. J Headache Pain 14:13

4. Harris J (1943) Habituatory response decrement in the intact organism. Psychol Bull 40:385-422

5. Thompson RF, Spencer WA (1966) Habituation: a model phenomenon for the study of neuronal substrates of behavior. Psychol Rev 73:16-43

6. Groves PM, Thompson RF (1970) Habituation: a dual-process theory. Psychol Rev 77:419-450

7. Glanzman DL (2009) Habituation in Aplysia: the Cheshire cat of neurobiology. Neurobiol Learn Mem 92:147-154

8. Thompson R (2009) Habituation: a history. Neurobiol Learn Mem 92:127-134

9. Mesulam MM (1990) Large-scale neurocognitive networks and distributed processing for attention, language, and memory. Ann Neurol 28:597-613

10. Kandel ER, Tauc L (1965) Heterosynaptic facilitation in neurones of the abdominal ganglion of Aplysia depilans. J Physiol 181:1-27

11. Thompson R, Glanzman D (1976) Neural and behavioral mechanisms of habituation and sensitization. In: Tighe TJ, Leaton RN (ed) Habituation. Lawrence Erlbaum Associates, Hillsdale, New Jersey, pp 49-93

12. Rankin CH, Abrams T, Barry RJ, Bhatnagar S, Clayton DF, Colombo J, et al. (2009) Habituation revisited: an updated and revised description of the behavioral characteristics of habituation. Neurobiol Learn Mem 92:135-138

13. Woolf C (2011) Central sensitization: implications for the diagnosis and treatment of pain. Pain 152:S2-15

14. Woolf CJ, Wall PD (1986) Relative effectiveness of $C$ primary afferent fibers of different origins in evoking a prolonged facilitation of the flexor reflex in the rat. J Neurosci 6:1433-1442

15. Schoenen J, Bottin D, Hardy F, Gerard P (1991) Cephalic and extracephalic pressure pain thresholds in chronic tension-type headache. Pain 47:145-149

16. Burstein R, Cutrer MF, Yarnitsky D (2000) The development of cutaneous allodynia during a migraine attack clinical evidence for the sequential recruitment of spinal and supraspinal nociceptive neurons in migraine. Brain 123(Pt 8):1703-1709

17. Bigal ME, Ashina S, Burstein R, Reed ML, Buse D, Serrano D, et al. (2008) Prevalence and characteristics of allodynia in headache sufferers: a population study. Neurology 70:1525-1533

18. Filatova E, Latysheva N, Kurenkov A (2008) Evidence of persistent central sensitization in chronic headaches: a multi-method study. J Headache Pain 9:295-300

19. Lovati C, D'Amico D, Bertora P, Rosa S, Suardelli M, Mailland E, et al. (2008) Acute and interictal allodynia in patients with different headache forms: an Italian pilot study. Headache 48:272-277
20. Lambert G, Truong L, Zagami A (2011) Effect of cortical spreading depression on basal and evoked traffic in the trigeminovascular sensory system. Cephalalgia 31:1439-1451

21. Goadsby P, Akerman S (2012) The trigeminovascular system does not require a peripheral sensory input to be activated-migraine is a central disorder. Focus on 'Effect of cortical spreading depression on basal and evoked traffic in the trigeminovascular sensory system'. Cephalalgia 32:3-5

22. Schoenen J, Maertens A, Timsit-Berthier M, et al. (1985) Contingent negative variation (CNV) as a diagnostic and physiopathologic tool in headache patients. In: Rose FC (ed) Migraine. Clinical and Research Advances, Basel: Karger, pp 17-25

23. Maertens de Noordhout A, Timsit-Berthier M, Timsit M, Schoenen J (1986) Contingent negative variation in headache. Ann Neurol 19:78-80

24. Kropp P, Gerber WD (1993) Contingent negative variation-findings and perspectives in migraine. Cephalalgia 13:33-36

25. Schoenen J, Timsit-Berthier M (1993) Contingent negative variation: methods and potential interest in headache. Cephalalgia 13:28-32

26. Kropp P, Gerber WD (1995) Contingent negative variation during migraine attack and interval: evidence for normalization of slow cortical potentials during the attack. Cephalalgia 15:123-128

27. Kropp P, Gerber WD (1993) Is increased amplitude of contingent negative variation in migraine due to cortical hyperactivity or to reduced habituation? Cephalalgia 13:37-41

28. Siniatchkin M, Gerber WD, Kropp P, Voznesenskaya T, Vein AM (2000) Are the periodic changes of neurophysiological parameters during the pain-free interval in migraine related to abnormal orienting activity? Cephalalgia 20:20-29

29. Siniatchkin M, Kropp P, Gerber WD (2001) Contingent negative variation in subjects at risk for migraine without aura. Pain 94:159-167

30. Siniatchkin M, Averkina N, Andrasik F, Stephani U, Gerber WD (2006) Neurophysiological reactivity before a migraine attack. Neurosci Lett 400:121-124

31. Siniatchkin M, Averkina N, Gerber WD (2006) Relationship between precipitating agents and neurophysiological abnormalities in migraine. Cephalalgia 26:457-465

32. Siniatchkin M, Andrasik F, Kropp P, Niederberger U, Strenge H, Averkina N, et al. (2007) Central mechanisms of controlled-release metoprolol in migraine: a double-blind, placebo-controlled study. Cephalalgia 27:1024-1032

33. Siniatchkin M, Gerber-von M, Darabaneanu S, Petermann F, Stephani U, Gerber W, et al. (2011) Behavioural treatment programme contributes to normalization of contingent negative variation in children with migraine. Cephalalgia 31:562-572

34. Evers S, Bauer B, Suhr B, Husstedt I, Grotemeyer K (1997) Cognitive processing in primary headache: a study on event-related potentials. Neurology 48:108-113

35. Evers S, Quibeldey F, Grotemeyer KH, Suhr B, Husstedt IW (1999) Dynamic changes of cognitive habituation and serotonin metabolism during the migraine interval. Cephalalgia 19:485-491

36. Wang W, Schoenen J, Timsit-Berthier M (1995) Cognitive functions in migraine without aura between attacks: a psychophysiological approach using the "oddball" paradigm. Neurophysiol Clin 25:3-11

37. Wang W, Schoenen J (1998) Interictal potentiation of passive "oddball" auditory event-related potentials in migraine. Cephalalgia 18:261-265

38. Siniatchkin M, Kropp P, Gerber WD (2003) What kind of habituation is impaired in migraine patients? Cephalalgia 23:511-518

39. Schoenen J, Wang W, Albert A, Delwaide P (1995) Potentiation instead of habituation characterizes visual evoked potentials in migraine patients between attacks. Eur J Neurol 2:115-122

40. Afra J, Cecchini AP, De Pasqua V, Albert A, Schoenen J (1998) Visual evoked potentials during long periods of pattern-reversal stimulation in migraine. Brain 121(Pt 2):233-241

41. Wang W, Wang GP, Ding XL, Wang YH (1999) Personality and response to repeated visual stimulation in migraine and tension-type headaches. Cephalalgia 19:718-724

42. Bohotin V, Fumal A, Vandenheede M, Gérard P, Bohotin C, Maertens de Noordhout A, et al. (2002) Effects of repetitive transcranial magnetic stimulation on visual evoked potentials in migraine. Brain 125:912-922

43. Ozkul Y, Bozlar S (2002) Effects of fluoxetine on habituation of pattern reversal visually evoked potentials in migraine prophylaxis. Headache 42:582-587 
44. Di Clemente L, Coppola G, Magis D, Fumal A, De Pasqua V, Schoenen J, et al. (2005) Nociceptive blink reflex and visual evoked potential habituations are correlated in migraine. Headache 45:1388-1393

45. Fumal A, Coppola G, Bohotin V, Gérardy PY, Seidel L, Donneau AF, et al. (2006) Induction of long-lasting changes of visual cortex excitability by five daily sessions of repetitive transcranial magnetic stimulation (rTMS) in healthy volunteers and migraine patients. Cephalalgia 26:143-149

46. Coppola G, Ambrosini A, Di Clemente L, Magis D, Fumal A, Gérard P, et al. (2007) Interictal abnormalities of gamma band activity in visual evoked responses in migraine: an indication of thalamocortical dysrhythmia? Cephalalgia 27:1360-1367

47. Coppola G, Currà A, Serrao M, Di Lorenzo C, Gorini M, Porretta E, et al. (2010) Lack of cold pressor test-induced effect on visual-evoked potentials in migraine. J Headache Pain 11:115-121

48. Coppola G, Currà A, Sava SL, Alibardi A, Parisi V, Pierelli F, et al. (2010) Changes in visual-evoked potential habituation induced by hyperventilation in migraine. J Headache Pain 11:497-503

49. Coppola G, Crémers J, Gérard P, Pierelli F, Schoenen J (2011) Effects of light deprivation on visual evoked potentials in migraine without aura. BMC Neurol 11:91

50. Chen WT, Wang SJ, Fuh JL, Lin CP, Ko YC, Lin YY, et al. (2009) Peri-ictal normalization of visual cortex excitability in migraine: an MEG study. Cephalalgia 29:1202-1211

51. Chen WT, Lin YY, Fuh JL, Hämäläinen MS, Ko YC, Wang SJ, et al. (2011) Sustained visual cortex hyperexcitability in migraine with persistent visual aura. Brain 134:2387-2395

52. Chen W, Wang S, Fuh J, Lin C, Ko Y, Lin Y, et al. (2011) Persistent ictal-like visual cortical excitability in chronic migraine. Pain 152:254-258

53. Ozkul Y, Uckardes A (2002) Median nerve somatosensory evoked potentials in migraine. Eur J Neurol 9:227-232

54. Coppola G, Currà A, Di Lorenzo C, Parisi V, Gorini M, Sava SL, et al. (2010) Abnormal cortical responses to somatosensory stimulation in medicationoveruse headache. BMC Neurol 10:126

55. Coppola G, De P, Pierelli F, Schoenen J (2012) Effects of repetitive transcranial magnetic stimulation on somatosensory evoked potentials and high frequency oscillations in migraine. Cephalalgia 32:700-709

56. Wang W, Timsit-Berthier M, Schoenen J (1996) Intensity dependence of auditory evoked potentials is pronounced in migraine: an indication of cortical potentiation and low serotonergic neurotransmission? Neurology 46:1404-1409

57. Ambrosini A, Rossi P, De Pasqua V, Pierelli F, Schoenen J (2003) Lack of habituation causes high intensity dependence of auditory evoked cortical potentials in migraine. Brain 126:2009-2015

58. Sand T, Zhitniy N, White LR, Stovner $\sqcup$ (2008) Brainstem auditory-evoked potential habituation and intensity-dependence related to serotonin metabolism in migraine: a longitudinal study. Clin Neurophysiol 119:1190-1200

59. Brodsky J, Mejico L, Giraud A, Woods C (2013) Impairment of habituation of the auditory brain stem response in migrainous vertigo. Ann Otol Rhinol Laryngol. 122:308-315

60. Katsarava Z, Giffin N, Diener HC, Kaube H (2003) Abnormal habituation of 'nociceptive' blink reflex in migraine-evidence for increased excitability of trigeminal nociception. Cephalalgia 23:814-819

61. De Marinis M, Pujia A, Natale L, D'arcangelo E, Accornero N (2003) Decreased habituation of the R2 component of the blink reflex in migraine patients. Clin Neurophysiol 114:889-893

62. Di Clemente L, Coppola G, Magis D, Fumal A, De Pasqua V, Di Piero V, et al. (2007) Interictal habituation deficit of the nociceptive blink reflex: an endophenotypic marker for presymptomatic migraine? Brain 130:765-770

63. Allena M, Magis D, De Pasqua V, Schoenen J, Bisdorff AR (2007) The vestibulo-collic reflex is abnormal in migraine. Cephalalgia 27:1150-1155

64. Roceanu A, Allena M, De Pasqua V, Bisdorff A, Schoenen J (2008) Abnormalities of the vestibulo-collic reflex are similar in migraineurs with and without vertigo. Cephalalgia 28:988-990

65. de Tommaso M, Libro G, Guido M, Losito L, Lamberti P, Livrea P, et al. (2005) Habituation of single CO2 laser-evoked responses during interictal phase of migraine. J Headache Pain 6:195-198

66. Valeriani M, de Tommaso M, Restuccia D, Le Pera D, Guido M, lannetti GD, et al. (2003) Reduced habituation to experimental pain in migraine patients: a $\mathrm{CO}(2)$ laser evoked potential study. Pain 105:57-64

67. de Tommaso M, Lo Sito L, Di Fruscolo O, Sardaro M, Pia Prudenzano M, Lamberti P, et al. (2005) Lack of habituation of nociceptive evoked responses and pain sensitivity during migraine attack. Clin Neurophysiol 116:1254-1264

68. de Tommaso M, Valeriani M, Sardaro M, Serpino C, Fruscolo OD, Vecchio E, et al. (2009) Pain perception and laser evoked potentials during menstrual cycle in migraine. J Headache Pain 10:423-429

69. Di Clemente L, Puledda F, Biasiotta A, Viganò A, Vicenzini E, Truini A, et al. (2013) Topiramate modulates habituation in migraine: evidences from nociceptive responses elicited by laser evoked potentials. J Headache Pain 14:25

70. Lev R, Granovsky Y, Yarnitsky D (2010) Orbitofrontal disinhibition of pain in migraine with aura: an interictal EEG-mapping study. Cephalalgia 30:910-918

71. Lev R, Granovsky Y, Yarnitsky D (2013) Enhanced Pain Expectation in Migraine: EEG-Based Evidence for Impaired Prefrontal Function. Headache 53:1054-1070

72. Qu C, Huo F, Huang F, Li Y, Tang J, Jia H, et al. (2008) The role of 5-HT receptor subtypes in the ventrolateral orbital cortex of 5-HT-induced antinociception in the rat. Neuroscience 152:487-494

73. Ferrari MD, Saxena PR (1993) On serotonin and migraine: a clinical and pharmacological review. Cephalalgia 13:151-165

74. Panconesi A (2008) Serotonin and migraine: a reconsideration of the central theory. J Headache Pain 9:267-276

75. Sakai Y, Dobson C, Diksic M, Aubé M, Hamel E (2008) Sumatriptan normalizes the migraine attack-related increase in brain serotonin synthesis. Neurology 70:431-439

76. Sándor PS, Afra J, Proietti-Cecchini A, Albert A, Schoenen J (1999) Familial influences on cortical evoked potentials in migraine. Neuroreport 10:1235-1238

77. Sand T, Vingen JV (2000) Visual, long-latency auditory and brainstem auditory evoked potentials in migraine: relation to pattern size, stimulus intensity, sound and light discomfort thresholds and pre-attack state. Cephalalgia 20:804-820

78. Judit A, Sándor PS, Schoenen J (2000) Habituation of visual and intensity dependence of auditory evoked cortical potentials tends to normalize just before and during the migraine attack. Cephalalgia 20:714-719

79. Kropp P, Gerber WD (1998) Prediction of migraine attacks using a slow cortical potential, the contingent negative variation. Neurosci Lett 257:73-76

80. Siniatchkin M, Kropp P, Gerber WD, Stephani U (2000) Migraine in childhood-are periodically occurring migraine attacks related to dynamic changes of cortical information processing? Neurosci Lett 279:1-4

81. Oelkers R, Grosser K, Lang E, Geisslinger G, Kobal G, Brune K, et al. (1999) Visual evoked potentials in migraine patients: alterations depend on pattern spatial frequency. Brain 122(Pt 6):1147-1155

82. Lang E, Kaltenhäuser M, Neundörfer B, Seidler S (2004) Hyperexcitability of the primary somatosensory cortex in migraine-a magnetoencephalographic study. Brain 127:2459-2469

83. Oelkers-Ax R, Parzer P, Resch F, Weisbrod M (2005) Maturation of early visual processing investigated by a pattern-reversal habituation paradigm is altered in migraine. Cephalalgia 25:280-289

84. Sand T, Zhitniy N, White LR, Stovner $L$ (2008) Visual evoked potential latency, amplitude and habituation in migraine: a longitudinal study. Clin Neurophysiol 119:1020-1027

85. Sand T, White L, Hagen K, Stovner L (2009) Visual evoked potential and spatial frequency in migraine: a longitudinal study. Acta Neurol Scand Suppl 189:33-37

86. Demarquay G, Caclin A, Brudon F, Fischer C, Morlet D (2011) Exacerbated attention orienting to auditory stimulation in migraine patients. Clin Neurophysiol 122:1755-1763

87. Omland P, Nilsen K, Uglem M, Gravdahl G, Linde M, Hagen K, et al. (2013) Visual Evoked Potentials in Interictal Migraine: No Confirmation of Abnormal Habituation. Headache 53:1071-1086

88. Coppola G, Parisi V, Di Lorenzo C, Serrao M, Magis D, Schoenen J, et al. (2013) Lateral inhibition in visual cortex of migraine patients between attacks. J Headache Pain 14:20

89. Lorenzo C, Coppola G, Currà A, Grieco G, Santorelli F, Lepre C, et al. (2012) Cortical response to somatosensory stimulation in medication overuse headache patients is influenced by angiotensin converting enzyme (ACE) I/ D genetic polymorphism. Cephalalgia 32:1189-1197

90. Restuccia D, Vollono C, Del P, Martucci L, Zanini S (2012) Somatosensory High Frequency Oscillations reflect clinical fluctuations in migraine. Clin Neurophysiol 123:2050-2056 
91. Restuccia D, Vollono C, Del P, Martucci L, Zanini S (2013) Different levels of cortical excitability reflect clinical fluctuations in migraine. Cephalalgia. doi:10.1177/0333102413482199

92. Noseda R, Kainz V, Jakubowski M, Gooley J, Saper C, Digre K, et al. (2010) A neural mechanism for exacerbation of headache by light. Nat Neurosci 13:239-245

93. Coppola G, Pierelli F, Schoenen J (2007) Is the cerebral cortex hyperexcitable or hyperresponsive in migraine? Cephalalgia 27:1427-1439

94. Aurora SK, Wilkinson F (2007) The brain is hyperexcitable in migraine. Cephalalgia 27:1442-1453

95. Brighina F, Palermo A, Fierro B (2009) Cortical inhibition and habituation to evoked potentials: relevance for pathophysiology of migraine. J Headache Pain 10:77-84

96. Viganò A, D'Elia T, Sava S, Auvé M, De P, Colosimo A, et al. (2013) Transcranial Direct Current Stimulation (tDCS) of the visual cortex: a proof-of -concept study based on interictal electrophysiological abnormalities in migraine. J Headache Pain 14:23

97. Coppola G, Vandenheede M, Di Clemente L, Ambrosini A, Fumal A, De Pasqua V, et al. (2005) Somatosensory evoked high-frequency oscillations reflecting thalamo-cortical activity are decreased in migraine patients between attacks. Brain 128:98-103

98. Sakuma K, Takeshima T, Ishizaki K, Nakashima K (2004) Somatosensory evoked high-frequency oscillations in migraine patients. Clin Neurophysiol 115:1857-1862

99. Ratliff F, Zemon V (1982) Some new methods for the analysis of lateral interactions that influence the visual evoked potential. Ann N Y Acad Sci 388:113-124

100. Zemon V, Ratliff F (1982) Visual evoked potentials: evidence for lateral interactions. Proc Natl Acad Sci U S A 79:5723-5726

101. Grose-Fifer J, Zemon V, Gordon J (1994) Temporal tuning and the development of lateral interactions in the human visual system. Invest Ophthalmol Vis Sci. 35:2999-3010

102. Baranyi A, Fehér O (1981) Synaptic facilitation requires paired activation of convergent pathways in the neocortex. Nature 290:413-415

103. Baranyi A, Szente MB (1987) Long-lasting potentiation of synaptic transmission requires postsynaptic modifications in the neocortex. Brain Res 423:378-384

104. Baranyi A, Szente MB, Woody CD (1991) Properties of associative longlasting potentiation induced by cellular conditioning in the motor cortex of conscious cats. Neuroscience 42:321-334

105. Pierelli F, lacovelli E, Bracaglia M, Serrao M, Coppola G (2013) Abnormal sensorimotor plasticity in migraine without aura patients. Pain. doi:10.1016/j. pain.2013.05.023

106. Kaube H, Katsarava Z, Przywara S, Drepper J, Ellrich J, Diener HC, et al. (2002) Acute migraine headache: possible sensitization of neurons in the spinal trigeminal nucleus? Neurology 58:1234-1238

107. de Tommaso M, Guido M, Libro G, Losito L, Sciruicchio V, Monetti C, et al. (2002) Abnormal brain processing of cutaneous pain in migraine patients during the attack. Neurosci Lett 333:29-32

108. de Tommaso M, Guido M, Libro G, Losito L, Difruscolo O, Puca F, et al. (2004) Topographic and dipolar analysis of laser-evoked potentials during migraine attack. Headache 44:947-960

109. de Tommaso M, Losito L, Libro G, Guido M, Di Fruscolo O, Sardaro M, et al. (2005) Effects of symptomatic treatments on cutaneous hyperalgesia and laser evoked potentials during migraine attack. Cephalalgia 25:359-368

110. Sand T, Zhitniy N, Nilsen K, Helde G, Hagen K, Stovner L, et al. (2008) Thermal pain thresholds are decreased in the migraine preattack phase. Eur J Neurol. 15:1199-1205

111. Schwedt T, Krauss M, Frey K, Gereau R (2011) Episodic and chronic migraineurs are hypersensitive to thermal stimuli between migraine attacks. Cephalalgia 31:6-12

112. Engstrøm M, Hagen K, Bjørk M, Stovner L, Gravdahl G, Stjern M, et al. (2013) Sleep quality, arousal and pain thresholds in migraineurs: a blinded controlled polysomnographic study. J Headache Pain 14:12

113. Bishop K, Holm J, Borowiak D, Wilson B (2001) Perceptions of pain in women with headache: a laboratory investigation of the influence of painrelated anxiety and fear. Headache 41:494-499

114. Weissman-Fogel I, Sprecher E, Granovsky Y, Yarnitsky D (2003) Repeated noxious stimulation of the skin enhances cutaneous pain perception of migraine patients in-between attacks: clinical evidence for continuous sub- threshold increase in membrane excitability of central trigeminovascular neurons. Pain 104:693-700

115. Sandrini G, Rossi P, Milanov I, Serrao M, Cecchini AP, Nappi G, et al. (2006) Abnormal modulatory influence of diffuse noxious inhibitory controls in migraine and chronic tension-type headache patients. Cephalalgia 26:782-789

116. de Tommaso M, Valeriani M, Guido M, Libro G, Specchio LM, Tonali P, et al. (2003) Abnormal brain processing of cutaneous pain in patients with chronic migraine. Pain 101:25-32

117. de Tommaso M, Losito L, Difruscolo O, Libro G, Guido M, Livrea P, et al. (2005) Changes in cortical processing of pain in chronic migraine. Headache 45:1208-1218

118. Ferraro D, Vollono C, Miliucci R, Virdis D, De A, Pazzaglia C, et al. (2012) Habituation to pain in "medication overuse headache": a CO2 laser-evoked potential study. Headache 52:792-807

119. Ayzenberg I, Obermann M, Nyhuis P, Gastpar M, Limmroth V, Diener HC, et al. (2006) Central sensitization of the trigeminal and somatic nociceptive systems in medication overuse headache mainly involves cerebral supraspinal structures. Cephalalgia 26:1106-1114

120. Perrotta A, Serrao M, Sandrini G, Burstein R, Sances G, Rossi P, et al. (2010) Sensitisation of spinal cord pain processing in medication overuse headache involves supraspinal pain control. Cephalalgia 30:272-284

121. Perrotta A, Arce-Leal N, Tassorelli C, Gasperi V, Sances G, Blandini F, et al. (2012) Acute reduction of anandamide-hydrolase (FAAH) activity is coupled with a reduction of nociceptive pathways facilitation in medication-overuse headache subjects after withdrawal treatment. Headache 52:1350-1361

122. Demirci S, Savas S (2002) The auditory event related potentials in episodic and chronic pain sufferers. Eur J Pain 6:239-244

123. Valeriani M, Galli F, Tarantino S, Graceffa D, Pignata E, Miliucci R, et al. (2009) Correlation between abnormal brain excitability and emotional symptomatology in paediatric migraine. Cephalalgia 29:204-213

124. Ozkul Y, Ay H (2007) Habituation of sympathetic skin response in migraine and tension type headache. Auton Neurosci 134:81-84

125. Sand T, Zwart J (1994) The blink reflex in chronic tension-type headache, migraine, and cervicogenic headache. Cephalalgia 14:447-450

126. Avramidis T, Podikoglou D, Anastasopoulos I, Koutroumanidis M, Papadimitriou A (1998) Blink reflex in migraine and tension-type headache. Headache 38:691-696

127. Aktekin B, Yaltkaya K, Ozkaynak S, Oguz Y (2001) Recovery cycle of the blink reflex and exteroceptive suppression of temporalis muscle activity in migraine and tension-type headache. Headache 41:142-149

128. Sand T, Møll-Nilsen B, Zwart J (2006) Blink reflex R2 amplitudes in cervicogenic headache, chronic tension-type headache and migraine. Cephalalgia 26:1186-1191

129. Nardone R, Tezzon F (2003) Short latency trigemino-sternocleidomastoid response in patients with migraine. J Neurol 250:725-732

130. Nardone R, Tezzon F (2003) The trigemino-cervical reflex in tension-type headache. Eur J Neurol 10:307-312

131. Milanov I, Bogdanova D (2003) Trigemino-cervical reflex in patients with headache. Cephalalgia 23:35-38

132. Peddireddy A, Wang K, Svensson P, Arendt-Nielsen L (2009) Blink reflexes in chronic tension-type headache patients and healthy controls. Clin Neurophysiol 120:1711-1716

133. Pielsticker A, Haag G, Zaudig M, Lautenbacher S (2005) Impairment of pain inhibition in chronic tension-type headache. Pain 118:215-223

134. Göbel H, Weigle L, Kropp P, Soyka D (1992) Pain sensitivity and pain reactivity of pericranial muscles in migraine and tension-type headache. Cephalalgia 12:142-151

135. Jensen R, Rasmussen B, Pedersen B, Olesen J (1993) Muscle tenderness and pressure pain thresholds in headache. A population study. Pain 52:193-199

136. Bendtsen L, Jensen R, Olesen J (1996) Decreased pain detection and tolerance thresholds in chronic tension-type headache. Arch Neurol 53:373-376

137. Ashina S, Babenko L, Jensen R, Ashina M, Magerl W, Bendtsen L, et al. (2005) Increased muscular and cutaneous pain sensitivity in cephalic region in patients with chronic tension-type headache. Eur J Neurol 12:543-549

138. Bovim G (1992) Cervicogenic headache, migraine, and tension-type headache. Pressure-pain threshold measurements. Pain 51:169-173

139. Metsahonkala L, Anttila P, Laimi K, Aromaa M, Helenius H, Mikkelsson M, et al. (2006) Extracephalic tenderness and pressure pain threshold in children with headache. Eur J Pain 10:581-585 
140. Fernández-de-las-Peñas C, Madeleine P, Caminero A, Cuadrado M, ArendtNielsen L, Pareja J, et al. (2010) Generalized neck-shoulder hyperalgesia in chronic tension-type headache and unilateral migraine assessed by pressure pain sensitivity topographical maps of the trapezius muscle. Cephalalgia 30:77-86

141. Cathcart S, Winefield A, Lushington K, Rolan P (2010) Noxious inhibition of temporal summation is impaired in chronic tension-type headache. Headache 50:403-412

142. Ashina S, Bendtsen L, Ashina M, Magerl W, Jensen R (2006) Generalized hyperalgesia in patients with chronic tension-type headache. Cephalalgia 26:940-948

143. de Tommaso M, Libro G, Guido M, Sciruicchio V, Losito L, Puca F, et al. (2003) Heat pain thresholds and cerebral event-related potentials following painful $\mathrm{CO} 2$ laser stimulation in chronic tension-type headache. Pain 104:111-119

144. lacovelli E, Coppola G, Tinelli E, Pierelli F, Bianco F (2012) Neuroimaging in cluster headache and other trigeminal autonomic cephalalgias. J Headache Pain 13:11-20

145. Evers S, Bauer B, Suhr B, Voss H, Frese A, Husstedt I, et al. (1999) Cognitive processing is involved in cluster headache but not in chronic paroxysmal hemicrania. Neurology 53:357-363

146. Formisano R, Cerbo R, Ricci M, Agostino R, Cesarino F, Cruccu G, et al. (1987) Blink reflex in cluster headache. Cephalalgia 7:353-354

147. Perrotta A, Serrao M, Sandrini G, Bogdanova D, Tassorelli C, Bartolo M, et al. (2008) Reduced habituation of trigeminal reflexes in patients with episodic cluster headache during cluster period. Cephalalgia 28:950-959

148. Coppola G, Di Lorenzo C, Bracaglia M, Porretta E, Pierelli F (2011) Nociceptive blink reflex habituation in episodic cluster headache patients during cluster period. Cephalalgia 31:ScS2-ScS1

149. Holle D, Zillessen S, Gaul C, Naegel S, Kaube H, Diener H, et al. (2012) Habituation of the nociceptive blink reflex in episodic and chronic cluster headache. Cephalalgia 32:998-1004

150. Pavesi G, Granella F, Brambilla S, Medici D, Mancia D, Manzoni G, et al. (1987) Blink reflex in cluster headache:evidence of a trigeminal system disfunction. Cephalalgia 6:100-102

151. Raudino F (1990) The blink reflex in cluster headache. Headache 30:584-585

152. Lozza A, Schoenen J, Delwaide PJ (1997) Inhibition of the blink reflex R2 component after supraorbital and index finger stimulations is reduced in cluster headache: an indication for both segmental and suprasegmental dysfunction? Pain 71:81-88

153. Sprenger $T$, Willoch $F$, Miederer M, Schindler F, Valet M, Berthele A, et al. (2006) Opioidergic changes in the pineal gland and hypothalamus in cluster headache: a ligand PET study. Neurology 66:1108-1110

154. Magis D, Bruno M, Fumal A, Gérardy P, Hustinx R, Laureys S, et al. (2011) Central modulation in cluster headache patients treated with occipital nerve stimulation: an FDG-PET study. BMC Neurol 11:25

155. Sandrini G, Alfonsi E, Ruiz L, Pavesi G, Micieli G, Manzoni G, et al. (1991) Impairment of corneal pain perception in cluster headache. Pain 47:299-304

156. Bono G, Antonaci F, Sandrini G, Pucci E, Rossi F, Nappi G, et al. (1996) Pain pressure threshold in cluster headache patients. Cephalalgia 16:62-66

157. Sandrini G, Antonaci F, Lanfranchi S, Milanov I, Danilov A, Nappi G, et al. (2000) Asymmetrical reduction of the nociceptive flexion reflex threshold in cluster headache. Cephalalgia 20:647-652

158. Nappi G, Sandrini G, Alfonsi E, Cecchini A, Micieli G, Moglia A, et al. (2002) Impaired circadian rhythmicity of nociceptive reflex threshold in cluster headache. Headache 42:125-131

159. Perrotta A, Serrao M, Ambrosini A, Bolla M, Coppola G, Sandrini G, et al. (2013) Facilitated temporal processing of pain and defective supraspinal control of pain in cluster headache. Pain 154:1325-1332

160. Procacci P, Zoppi M, Maresca M, Zamponi A, Fanciullacci M, Sicuteri F, et al. (1989) Lateralisation of pain in cluster headache. Pain 38:275-278

161. Ladda J, Straube A, Förderreuther S, Krause P, Eggert T (2006) Quantitative sensory testing in cluster headache: increased sensory thresholds. Cephalalgia 26:1043-1050

162. Ellrich J, Ristic D, Yekta S (2006) Impaired thermal perception in cluster headache. J Neurol 253:1292-1299

163. Antonaci F, Sandrini G, Danilov A, Sand T (1994) Neurophysiological studies in chronic paroxysmal hemicrania and hemicrania continua. Headache 34:479-483

164. Palmieri A (2006) Chronic cluster headache responsive to pramipexole. Cephalalgia 26:761-762
165. Di Lorenzo C, Coppola G, Pierelli F (2013) A case of cluster headache treated with rotigotine: Clinical and neurophysiological correlates. Cephalalgia. doi:10.1177/0333102413490346

166. Malick A, Strassman R, Burstein R (2000) Trigeminohypothalamic and reticulohypothalamic tract neurons in the upper cervical spinal cord and caudal medulla of the rat. J Neurophysiol 84:2078-2112

167. Schoenen J, Jensen R, Lantéri-Minet M, Láinez M, Gaul C, Goodman A, et al. (2013) Stimulation of the sphenopalatine ganglion (SPG) for cluster headache treatment. Pathway $\mathrm{CH}-1$ : A randomized, sham-controlled study. Cephalalgia 33:816-830

168. Schoenen J, Vandersmissen B, Jeangette S, Herroelen L, Vandenheede M Gérard P, et al. (2013) Migraine prevention with a supraorbital transcutaneous stimulator: a randomized controlled trial. Neurology 80:697-704

\section{doi:10.1186/1129-2377-14-65}

Cite this article as: Coppola et al.: Habituation and sensitization in primary headaches. The Journal of Headache and Pain 2013 14:65.

\section{Submit your manuscript to a SpringerOpen ${ }^{\odot}$ journal and benefit from:}

- Convenient online submission

- Rigorous peer review

- Immediate publication on acceptance

- Open access: articles freely available online

- High visibility within the field

- Retaining the copyright to your article

Submit your next manuscript at $>$ springeropen.com 